\title{
Semen parameters on the intracytoplasmic sperm injection day: Predictive values and cutoff thresholds of success
}

\author{
Alaa El din-Abdel Aal Moubasher ', Emad Abdelrehim Taha', Ehab Mohamed Elnashar², Ahmed Abdel Aal Abdel Maged', \\ Asmaa Mohamed Zahran ${ }^{3}$, Heba Hassan Sayed ${ }^{1}$, Hisham Diab Gaber ${ }^{1}$ \\ Departments of 'Dermatology, Venereology and Andrology and ${ }^{2}$ Obstetrics and Gynecology, Faculty of Medicine, Assiut University, Assiut; \\ ${ }^{3}$ Department of Clinical Pathology, South Egypt Cancer Institute, Assiut University, Assiut, Egypt
}

Objective: This study was conducted to investigate the relationship of semen parameters in samples used for intracytoplasmic sperm injection (ICSI) with fertilization and pregnancy rates in infertile couples.

Methods: In this prospective study of Infertile couples with male factor infertility that had undergone ICSI, fractions of the same semen samples obtained for microinjection (to ensure the best predictability) were evaluated to determine the semen parameters and sperm DNA fragmentation index (DFI) on the day of oocyte recovery.

Results: In total, 120 couples completed the study and were subdivided into fertilized ( $n=87)$ and non-fertilized couples $(n=33)$. The fertilized couples were further classified into pregnant $(n=48)$ and non-pregnant $(n=39)$ couples. Compared to non-fertilized and non-pregnant couples, fertilized and pregnant couples showed statistically significantly higher sperm viability and percentage of normal sperm morphology, as well as significantly lower sperm DFI values. A receiver operating characteristic curve analysis of data from the $120 \mathrm{ICSI}$ cycles showed that sperm viability, normal sperm morphology percentages, and sperm DFI were significant prognostic indicators of fertilization at cutoff values of $40 \%, 7 \%$, and $46 \%$, respectively. A sperm DFI of $46 \%$ showed sensitivity and specificity of $95 \%$ and $90 \%$, respectively, for predicting fertilization, and no clinical pregnancies occurred in couples with a sperm DFI above $46 \%$.

Conclusion: Semen parameters from the ICSI day sample, especially sperm viability, normal morphology, and DFI, had an impact on fertilization and pregnancy outcomes in ICSI cycles.

Keywords: Intracytoplasmic sperm injection; Fertilization; Pregnancy rate; Semen; Sperm DNA fragmentation index

\section{Introduction}

The introduction of intracytoplasmic sperm injection (ICSI) has been heralded as one of the major breakthroughs in the field of reproductive medicine. Since the first live births with ICSI were report-

Received: July 30, 2020 · Revised: September 16, 2020 · Accepted: October 14, 2020 Corresponding author: Hisham D Gaber

Department of Dermatology, Venereology and Andrology, Faculty of Medicine, Assiut University, Assiut 17515, Egypt

Tel: +20-88-2340680 Fax: +20-88-2332278 E-mail: hishamdiab@aun.edu.eg

This is an Open Access article distributed under the terms of the Creative Commons Attribution Non-Commercial License (http://creativecommons.org/licenses/by-nc/4.0/) which permits unrestricted non-commercial use, distribution, and reproduction in any medium, provided the original work is properly cited. ed in 1992, this technique has been deployed as a powerful tool to treat almost all forms of male infertility, as well as to overcome fertilization failure. ICSI, in conjunction with in vitro fertilization (IVF), has been integral in millions of advanced reproductive treatments, resulting in the birth of over 5 million babies so far [1].

Following several refinements, ICSI has become a powerful tool for overcoming suboptimal semen parameters and fertilization defects, thereby allowing infertile men to reproduce at rates that previously would have been impossible [2]. Since ICSI bypasses many steps of the normal fertilization process, conventional sperm parameters such as morphology, motility, and viability have received little attention regarding their relationships with ICSI outcomes, with some 
conflicts and no consensus on cutoff values predictive of successful ICSI outcomes [3].

More importantly, sperm DNA parameters are recognized as objective, independent measures of sperm quality that may have better diagnostic and prognostic capabilities than standard sperm parameters, especially with assisted reproductive techniques [4]. Although research into the importance of sperm DNA fragmentation as a predictive factor for ICSI outcome has been conducted for more than 10 years, there is lack of consensus among studies about its precise role in predicting ICSI outcomes in terms of fertilization, embryo development, pregnancy, miscarriage, and live birth [5]. These inconsistencies may be attributed to study design, the use of different assays of sperm DNA fragmentation, and a lack of standardization.

Another important limitation is that the great majority of these studies, with few exceptions, obtained semen samples before assigning the studied men to ICSI cycles, rather than testing the sample obtained and used on the day of ICSI [6]. Additionally, most of these studies measured ICSI outcomes in terms of fertilization and chemical pregnancy rates, with no comment on clinical pregnancy [7]. Therefore, it is a pressing necessity to evaluate the effect of conventional semen parameters and sperm DNA fragmentation of the semen sample used for microinjection on ICSI outcomes in terms of fertilization, embryo quality, and clinical pregnancy.

\section{Methods}

This prospective controlled study was conducted at the Andrology Unit of the Dermatology, Venereology and Andrology Department, Assiut University Hospitals, in collaboration with the assisted conception unit at a women's health hospital and the Department of Clinical Pathology at South Egypt Cancer Institute, in the period from April 2018 to December 2019. The study was approved by the Institutional Review Board of Assiut University Hospital (IRB No. 17200054) and all patients signed an informed consent form. Privacy and confidentiality of all data were assured.

The study included infertile couples who presented to the assisted conception unit at a women's health hospital for ICSI procedure based on the diagnosis of male factor infertility with normal female partners. We excluded men taking medications affecting spermatogenesis or who had pyospermia, clinical varicocele, systemic diseases, a history of cryptorchidism, or azoospermia. Men with inadequate semen volume on the ICSI day were also excluded. We excluded female partners aged $>38$ years or with any gynecological diseases that could affect oocyte quality or endometrial receptivity, such as endometriosis, adenomyosis, pelvic inflammatory disease, pyosalpinx, or subendometrial fibroids, or systemic diseases that may impair reproductive capacity, such as hepatic, renal, or endocrine con- ditions, including diabetes mellitus and autoimmune diseases.

All female patients received ovarian stimulation using a standard luteal down-regulation regimen (long protocol) or a flare-up short regimen (short protocol) [8]. On the day of oocyte retrieval, semen samples were obtained from the male partners by masturbation. After complete liquefaction in a $37^{\circ} \mathrm{C}$ incubator, a small fraction $(0.5$ $\mathrm{mL}$ ) was taken from the ICSI sample for evaluation according to the World Health Organization (WHO) 2010 criteria [9] and sperm DNA fragmentation assessment, and the rest was used for microinjection of the selected sperm into retrieved oocytes according to the procedure reported by Palermo et al. [10].

\section{Sperm DNA fragmentation assessment}

Sperm DNA fragmentation was assessed using flow cytometry (Beckman Coulter, Fullerton, CA, USA) based on the fluorescence emission from individual spermatozoa stained with propidium iodide (PI) and excitation with a 488-nm argon laser. Flow cytometric detection of sperm DNA chromatin damage was carried out according to the method described by Martinez-Soto et al. [11]. A 100- $\mu \mathrm{L}$ fraction of the semen sample was diluted with phosphate-buffered saline to $2 \times 10^{6} \mathrm{sperm} / \mathrm{mL}$. Fifty microliters of the semen sample was directly stained with $50 \mu \mathrm{g} / \mathrm{mL}$ PI, using the cycle test kit (Becton Dickinson Biosciences, Franklin Lakes, NJ, USA) and analyzed immediately by FACSCalibur flowcytometry with Cell Quest software (Becton Dickinson Biosciences). Ten thousand events were measured for each specimen; this permitted the state of condensation of the sperm chromatin to be analyzed, as DNA condensation is directly related to PI uptake. The percentage of sperm cells with DNA damage was automatically calculated and the result was expressed as the sperm DNA fragmentation index (DFI).

\section{Fertilization assessment}

The oocytes were assessed for fertilization at 16-18 hours after the microinjection. Fertilization was confirmed to be normal if two pronuclei and two polar bodies were identified, and pronuclei size and position, as well as the size, distribution, and number of nucleoli, were evaluated. Oocytes without obvious pronuclei were considered unfertilized. Oocytes with a single pronucleus or more than two pronuclei were cancelled due to abnormal fertilization. The percentage of fertilization was calculated as the number of fertilized oocytes divided by the total number of injected metaphase II oocytes [12]. The included couples were classified according to the fertilization rate into fertilized couples versus non-fertilized couples.

\section{Embryo assessment and transfer}

Embryos were assessed according to their number, symmetry, blastomeres, type, and percentage of fragmentation according to 
the Istanbul consensus of embryo assessment [13]. Fresh embryo transfer was performed on day 3 or 5 after oocyte retrieval using the best-quality embryos among a cohort of resultant embryos. The grading criteria were as follows: grade I or good embryo quality: $<10 \%$ fragmentation with equal-sized cells and no multinucleation; grade II or fair embryo quality: 10\%-25\% fragmentation with equalsized cells and no evidence of multinucleation; and grade III or poor embryo quality: severe fragmentation ( $>25 \%$ ) with unequal-sized cells and evidence of multinucleation. No more than three surviving embryos were transferred into the uterine cavity. The extra embryos were cryopreserved for subsequent embryo transfers. The luteal phase was routinely supported with progesterone $(40-60 \mathrm{mg}$, intramuscular) per day for 14 days and continued for another 4 weeks if pregnancy was established.

\section{Pregnancy determination and follow-up}

Serum human chorionic gonadotropin levels were measured 14 days after embryo transfer. Clinical pregnancy was confirmed by observing a fetal heartbeat in the uterine cavity on ultrasound 4 weeks after embryo transfer. All pregnant women were followed up regularly in the prenatal clinic every 4 weeks until the 28th week of pregnancy. After the 28th week, the follow-up became every 2 weeks until the 36th week, and then, once a week until the end of pregnancy and delivery of the babies. On this basis, the live birth rate was analyzed.

\section{Statistical analysis}

Data entry and data analysis were done using IBM SPSS ver. 22 (IBM Corp., Armonk, NY, USA). Data were presented as number, percentage, mean, median and standard deviation. The chi-square test was used to compare qualitative variables.

The Student $t$-test was used to compare quantitative variables between groups for parametric data, while the Mann-Whitney test was used for non-parametric data. Spearman correlation coefficients were calculated to measure correlations between quantitative variables. MedCalc (Ostend, Belgium) was used to calculate sensitivity, specificity, positive and negative predictive values, and receiver operating characteristic (ROC) curves. For all tests, $p$-values $<0.05$ were considered to indicate statistical significance.

\section{Results}

The study initially included 134 couples, of whom 14 were excluded due to either inadequate semen samples or cancellation as a result of ovarian hyperstimulation or abnormal fertilization. The remaining 120 couples completed the study and were classified according to fertilization into fertilized $(n=87)$ and non-fertilized $(n=33)$ groups. Among the included couples, 50 were diagnosed with idiopathic male factor infertility, 40 were diagnosed with bilateral tubal blockage, and 30 had unexplained infertility. There were no significant differences in the mean ages of the male partners ( $35.27 \pm 4.78$ vs. $34.98 \pm 3.5$ years) and female partners ( $28.55 \pm 3.51$ vs. $29.13 \pm 3.24$ years) between the fertilized and non-fertilized groups. The mean infertility duration was not significantly between the two groups $(5.93 \pm 1.38$ years for the fertilized group vs. $6.83 \pm 2.61$ years for the non-fertilized group). There was also no significant difference in the mean retrieved oocyte number between the two groups ( $8.36 \pm 1.86$ for the fertilized group vs. $7.96 \pm 1.96$ for the non-fertilized group). The fertilization rate was $72 \%$ and the pregnancy rate was $55 \%$.

In the fertilized couples, statistically significantly higher values were found for total sperm motility, progressive motility, viability (using the hypo-osmotic swelling test [HOST]), and the percentage of normal morphology than in non-fertilized couples $(p=0.001$, $p=0.001, p=0.001$, and $p=0.001$, respectively), while the sperm DFI was significantly lower $(p=0.001)$. No statistically significant differences in semen volume or sperm concentration and count were noted between the fertilized and non-fertilized couples, as shown in Table 1.

The fertilized couples were further classified according to clinical pregnancy into pregnant $(n=48)$ and non-pregnant couples $(n=39)$. Significantly higher values were found for sperm viability and percentage of normal sperm morphology in the pregnant couples than in the non-pregnant couples ( $p=0.002$ and $p=0.045$, respectively). A significantly lower sperm DFI was found in the pregnant couples than in the non-pregnant couples $(p=0.001)$. No statistically significant differences were found in semen volume, total sperm motility, progressive motility, concentration, and count between pregnant and non-pregnant couples, as shown in Table 2.

According to the ROC curve analysis of data from the 120 ICSI cycles, sperm viability, normal sperm morphology percentage, and sperm DFI were statistically significant as a prognostic indicators of the fertilization rate, with areas under the curve (AUCs) of 0.745 , 0.770 , and 0.965 respectively (Figure 1). The values with the best ratio of sensitivity and specificity were evaluated and were found to be $40 \%, 7 \%$, and $46 \%$, respectively; these can be used as cutoff values for predicting fertilization. A pairwise comparison of the three ROC curves in Figure 1 showed significant differences between sperm DFI and sperm viability (difference between AUCs, $0.220 ; p=0.001$ ) and normal sperm morphology (difference between AUCs, 0.195; $p=0.001$ ). However, there was no significant difference between the ROC curves for sperm viability and normal morphology (difference between AUCs, $0.0252 ; p=0.54)$. Sperm DFI was also a statistically significant prognostic indicator of the clinical pregnancy rate, with 
Table 1. Comparison between semen parameters and sperm DFI\% of the fertilized and non-fertilized couples

\begin{tabular}{|c|c|c|c|}
\hline Variable & Fertilized couple $(n=87)$ & Non-fertilized couple $(n=33)$ & $p$-value \\
\hline Semen volume $(\mathrm{mL})^{\mathrm{a})}$ & & & 0.462 \\
\hline Mean \pm SD & $2.31 \pm 0.60$ & $2.18 \pm 0.72$ & \\
\hline Median (range) & $2.0(1.0-3.0)$ & $2.0(1.0-3.0)$ & \\
\hline Total motility (\%) & & & $0.001^{c}$ \\
\hline Mean \pm SD & $30.67 \pm 6.79$ & $22.00 \pm 5.51$ & \\
\hline Median (range) & $30.0(20.0-55.0)$ & $20.0(15.0-30.0)$ & \\
\hline Progressive motility $(\%)^{a}$ & & & $0.001^{c)}$ \\
\hline Mean \pm SD & $16.04 \pm 6.47$ & $8.33 \pm 2.40$ & \\
\hline Median (range) & $15.0(5.0-32.0)$ & $10.0(5.0-10.0)$ & \\
\hline Viability by HOST (\%) & & & $0.001^{c)}$ \\
\hline Mean \pm SD & $51.11 \pm 9.71$ & $41.33 \pm 8.60$ & \\
\hline Median (range) & $50.0(10.0-65.0)$ & $40.0(30.0-60.0)$ & \\
\hline Sperm concentration (million $/ \mathrm{mL}$ ) & & & $0.927^{c}$ \\
\hline Mean \pm SD & $13.87 \pm 6.15$ & $13.10 \pm 4.56$ & \\
\hline Median (range) & $10.0(8.0-38.0)$ & $10.0(5.0-20.0)$ & \\
\hline Sperm count (million/mL) & & & $0.315^{c)}$ \\
\hline Mean \pm SD & $31.04 \pm 13.24$ & $26.97 \pm 8.97$ & \\
\hline Median (range) & $30.0(10.0-76.0)$ & $30.0(10.0-40.0)$ & \\
\hline Normal sperm morphology by spermac stain (\%) & & & $0.001^{c)}$ \\
\hline Mean \pm SD & $5.60 \pm 3.27$ & $2.67 \pm 1.49$ & \\
\hline Median (range) & $4.0(2.0-10.0)$ & $2.0(2.0-10.0)$ & \\
\hline Sperm DFI (\%) & & & $0.001^{c}$ \\
\hline Mean \pm SD & $30.89 \pm 11.44$ & $59.18 \pm 10.38$ & \\
\hline Median (range) & $28.0(4.0-67.0)$ & $58.5(40.0-83.0)$ & \\
\hline
\end{tabular}

DFI, DNA fragmentation index; SD, standard deviation; HOST, hypo-osmotic swelling test.

${ }^{\text {a) }}$ Student $t$-test; ${ }^{\text {b) }}$ Mann-Whitney test; ${ }^{\text {c) }}$ Significant $p$-value $<0.05$.

an AUC of 0.788 . The value with the best ratio of sensitivity and specificity was evaluated and was found to be $33 \%$, which can be used as a cutoff value for predicting pregnancy (Supplementary Figure 1).

Using the cutoff value of DFI (46\%) for predicting fertilization according to the ROC curve (Figure 1), the patients were divided into two groups, as shown in Table 3. We compared the DFI groups according to the outcomes of the ICSI procedure and we found that the fertilization rate and good-quality embryo rate were significantly higher in the low-DFI group than in the high-DFI group $(p=0.001$ and $p=0.026$, respectively). No clinical pregnancy was achieved in patients with a sperm DFI higher than $46 \%$.

\section{Discussion}

Male infertility management has grown at a slower pace than female infertility management. Despite being revolutionized by ICSI, the success rate of the technique is much lower in patients with male infertility [14]. To improve the success rate, there is a continuing need for more research to unravel the mystery of male infertility parameters that may have predictive value for the different measures of ICSI outcomes [15].

The current study evaluated semen parameters and sperm DFI as predictors for ICSI outcome among 120 couples who were classified according to fertilization into fertilized and non-fertilized couples. In this study, statistically significantly higher values for total sperm motility, progressive sperm motility, sperm count, and the percentage of normal sperm morphology were found among fertilized couples than non-fertilized couples. Sperm viability, normal morphology, and DFI were significant prognostic factors of fertilization.

HOST has been shown to be an effective method for selecting live sperm for ICSI. In this study, sperm viability was significantly higher in the fertilized and pregnant couples than in the non-fertilized and non-pregnant couples. A cutoff level of $40 \%$ of sperm viability was found to be a predictor for the fertilization rate in subsequent analyses, with a sensitivity of $91 \%$ and a specificity of $52.5 \%$. These findings are in accordance with those of Charehjooy et al. [16], who reported a significantly higher percentage of fertilization, embryos that had good quality, implantation, and pregnancy rates in a group of infertile men undergoing ICSI cycles in which HOST was used as a guide for sperm selection. Other studies have also noted higher rates 
Table 2. Comparison between semen parameters and sperm DFI\% of the pregnant and non-pregnant couples

\begin{tabular}{|c|c|c|c|}
\hline Variable & Pregnant couple $(\mathrm{no}=48)$ & Non-pregnant couple $(\mathrm{no}=39)$ & $p$-value \\
\hline Semen volume $(\mathrm{mL})^{\mathrm{a})}$ & & & 0.586 \\
\hline Mean \pm SD & $2.36 \pm 0.57$ & $2.25 \pm 0.64$ & \\
\hline Median (range) & $2.0(1.0-3.0)$ & $2.0(1.0-3.0)$ & \\
\hline Total motility (\%) & & & 0.507 \\
\hline Mean \pm SD & $31.40 \pm 7.57$ & $29.75 \pm 5.73$ & \\
\hline Median (range) & $30.0(20.0-55.0)$ & $30.0(20.0-40.0)$ & \\
\hline Progressive motility (\%) ${ }^{\mathrm{a})}$ & & & 0.475 \\
\hline Mean \pm SD & $16.88 \pm 7.07$ & $15.00 \pm 5.62$ & \\
\hline Median (range) & $15.0(10.0-32.0)$ & $15.0(5.0-25.0)$ & \\
\hline Viability by HOST (\%) & & & $0.022^{c)}$ \\
\hline Mean \pm SD & $53.00 \pm 11.81$ & $48.75 \pm 5.59$ & \\
\hline Median (range) & $50.0(10.0-65.0)$ & $50.0(40.0-60.0)$ & \\
\hline Sperm concentration (million $/ \mathrm{mL}$ ) & & & $0.09^{c)}$ \\
\hline Mean \pm SD & $12.96 \pm 5.95$ & $13.25 \pm 5.67$ & \\
\hline Median (range) & $11.0(8.0-38.0)$ & $12.0(10.0-30.0)$ & \\
\hline Sperm count (million/ejaculate) $\left.{ }^{b}\right)$ & & & $0.058^{c)}$ \\
\hline Mean \pm SD & $28.08 \pm 12.36$ & $34.75 \pm 13.66$ & \\
\hline Median (range) & $30.0(10.0-76.0)$ & $33.0(15.0-60.0)$ & \\
\hline Normal sperm morphology by spermac stain (\%) & & & $0.045^{c}$ \\
\hline Mean \pm SD & $6.68 \pm 3.56$ & $4.25 \pm 2.31$ & \\
\hline Median (range) & $8.0(2.0-10.0)$ & $3.0(2.0-8.0)$ & \\
\hline Sperm DFI\% ${ }^{\mathrm{a})}$ & & & $0.001^{c}$ \\
\hline Mean \pm SD & $26.26 \pm 8.75$ & $38.60 \pm 11.35$ & \\
\hline Median (range) & $27.5(4.0-46.0)$ & $39.5(21.0-67.0)$ & \\
\hline
\end{tabular}

DFI, DNA fragmentation index; SD, standard deviation.

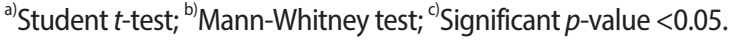

of fertilization in groups with higher sperm viability detected by HOST, although the results were not statistically significant [17].

A cutoff level (7\%) of normal sperm morphology percentage, with a sensitivity of $68.75 \%$ and a specificity of $77.5 \%$, was found to predict fertilization in ICSI procedures in our study. The WHO 2010 manual provides $4 \%$ as the lower limit of normal sperm morphology percentage; however, previous studies have reported different cutoff values for spontaneous and assisted conception [9]. Semen samples with an elevated proportion of abnormal sperm morphology usually have a high DFI, which may affect fertilization in response to ICSI [18].

Previous studies were contradictory and failed to identify a positive predictive value for routine semen parameters in ICSI outcomes, with no consensus on cutoff values predicting success $[12,19]$. This may be explained by differences among these studies in terms of the retrospective study design, etiology of infertility in the investigated infertile couples, or the edition of the WHO guidelines that was used.

Sperm DNA integrity is one of the most important objective parameters affecting ICSI outcomes, and it may even indicate male subfertility regardless of conventional semen parameters [20]. In our study, the DFI was significantly lower in both fertilized and pregnant couples than in non-fertilized and non-pregnant couples. Furthermore, sperm DFI was a significant prognostic indicator of both fertilization and pregnancy. This finding is in accordance with previous studies that reported negative correlations between the sperm DFI and fertilization, embryo grade, and pregnancy rates with ICSI cycles $[14,20]$. However, these results contradict the findings of some other studies that reported no correlation between the DFI and fertilization, embryo quality, or pregnancy rates in infertile patients undergoing ICSI treatment $[5,6,12,14,21]$. This may be due to a lack of standardization of sperm DNA fragmentation tests or the use of different methods of sperm selection techniques or different definitions of pregnancy outcomes. The variable ability of oocytes and early embryos to repair sperm DNA damage may be another explanation. Additionally, the paternal genome is switched on after the 4- to 8-cell stage, which further affects embryo development. In the stage of in vitro development, good-quality embryos may not necessarily develop to the blastocyst stage. Moreover, poor embryos might reach the blastocyst stage despite the higher level of DFI in the sperm used for fertilization [5,22].

In their recent study, Green and coauthors et al. [6] failed to find a 


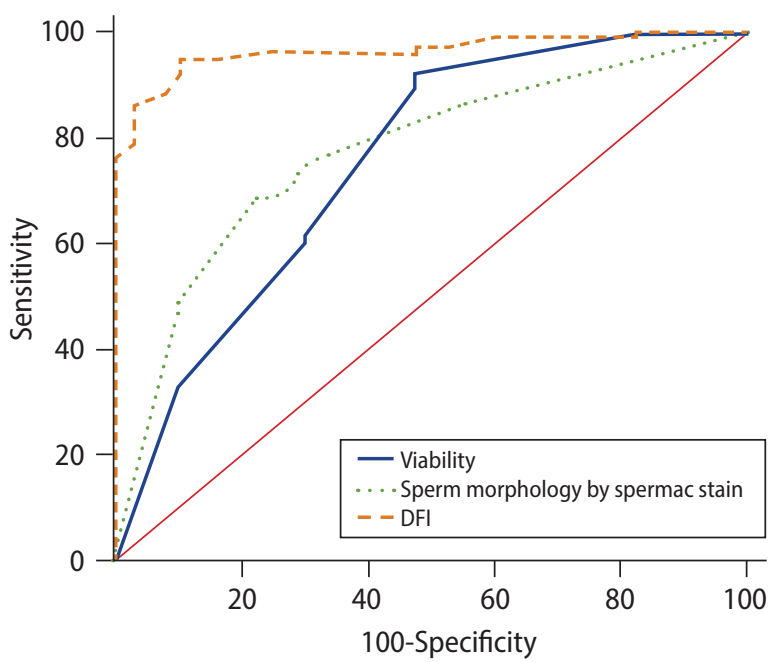

Figure 1. Receiver operating characteristic curve analysis. Sperm viability of the microinjection semen sample vs. fertilization rate; area under the curve (AUC) $=0.745$ ( $p<0.05$; accuracy, 78.33\%). The value with the best ratio of sensitivity $(91.25 \%)$ and specificity (52\%) was $40 \%$, which was used as the cutoff value for predicting fertilization in subsequent analyses. Normal sperm morphology percentage of the microinjection semen sample vs. fertilization rate; AUC $=0.770(p<0.05 ;$ accuracy, $71.67 \%)$. The value with the best ratio of sensitivity (68.75\%) and specificity (77.5\%) was $7 \%$, which was used as the cutoff value for predicting fertilization in subsequent analyses. Sperm DNA fragmentation index (DFI) vs. fertilization rate; AUC $=0.965(p<0.05 ;$ accuracy, $93.33 \%)$. The value with the best ratio of sensitivity (95\%) and specificity (90\%) was $46 \%$, which was used as the cutoff value for predicting fertilization in subsequent analyses.

difference in the fertilization, blastulation, implantation, pregnancy and miscarriage rates with ICSI between the low $(<15 \%)$ and high ( $>15 \%$ ) sperm DFI groups. Although the authors performed the analysis using semen samples obtained on the ICSI day, as we did, they used a different technique (sperm chromatin structure assay) for assessing sperm DNA damage and they assayed the embryos for aneuploidy before transfer using trophectoderm blastocyst biopsy with real-time polymerase chain reaction or next-generation sequencing.

In this study, a sperm DFI threshold of $46 \%$ was used as the cutoff value for predicting fertilization, with high sensitivity (95\%) and specificity (90\%). A threshold of 33\% was used as the cutoff value for predicting pregnancy, with a sensitivity of $84 \%$ and a specificity of $70 \%$. The fertilization rate was statistically significantly lower in men with a sperm DFI $>46 \%$ than in those with a DFI $\leq 46 \%$. Furthermore, a significantly higher good-quality embryo formation rate was found in the low-DFI group than in the high-DFI group. Previous studies used different cutoff values of DFI as assessed by various sperm DNA integrity assays, including 29\% [20], 22.3\% [23], and 15\% [6].
Table 3. Comparison between the different outcomes of ICSI in relation to cutoff value of sperm DFI

\begin{tabular}{|c|c|c|c|}
\hline Variable & $\begin{array}{c}\text { Low DFI }(\leq 46) \\
(n=87)\end{array}$ & $\begin{array}{l}\text { High DFI (>46) } \\
\quad(n=33)\end{array}$ & $p$-value \\
\hline Fertilization rate & & & $0.000^{\mathrm{a})}$ \\
\hline Yes & $83(95.4)$ & $4(12.1)$ & \\
\hline No & $4(4.6)$ & $29(87.9)$ & \\
\hline Embryo quality & & & $0.026^{\mathrm{a})}$ \\
\hline Good & $51(61.4)$ & 0 & \\
\hline Fair and poor & $32(38.6)$ & $4(100.0)$ & \\
\hline Pregnancy rate & & & - \\
\hline Yes & $48(57.8)$ & 0 & \\
\hline No & $35(42.2)$ & $4(100.0)$ & \\
\hline Abortion & & & - \\
\hline No abortion & $29(60.4)$ & - & \\
\hline First trimester abortion & $12(25)$ & - & \\
\hline Second trimester abortion & $7(14.6)$ & - & \\
\hline Live birth rate & & & - \\
\hline Yes & $29(60.4)$ & - & \\
\hline No & $19(39.6)$ & - & \\
\hline
\end{tabular}

Values are presented as number (\%).

ICSI, intracytoplasmic sperm injection; DFI, DNA fragmentation index.

Chi-square test. ${ }^{\text {a) }}$ Significant $p$-value $<0.05$.

We were not able to compare miscarriage or live birth rates between the low-DFI and high-DFI groups in our study, as no patient achieved pregnancy in the high DFI group. Some studies reported no statistically significant difference in the abortion rate or live birth rate between low- and high-DFI groups [6,20,24,25]. However, two meta-analyses found that sperm DNA damage was associated with a significantly increased risk of pregnancy loss after ICSI, and they recommended evaluating sperm DNA damage prior to ICSI $[26,27]$. Nonetheless, a well-designed meta-analysis failed to confirm the predictive value of sperm DFI for ICSI outcomes in terms of clinical pregnancy and pregnancy loss [28]. The different findings again can be attributed to different patient selection criteria and ICSI protocols among studies.

In conclusion, our observations emphasize the importance of sperm viability, normal morphology, and DNA integrity of the sample used for ICSI to enhance the likelihood of good outcomes. This necessitates proper management of male partners and the elimination of any treatable risk factors that could impair these parameters prior to enrollment in ICSI. Further studies are recommended to achieve a consensus on standardized protocols for sperm DNA evaluation and selection with ICSI, especially for patients affected by male factor infertility. 


\section{Conflict of interest}

No potential conflict of interest relevant to this article was reported.

\section{Acknowledgments}

We thank all patients participating in this investigation, as well as the medical and lab staff at the Faculty of Medicine, Assiut University, who helped us during this study.

\section{ORCID}

\section{Alaa El din-Abdel Aal Moubasher https://orcid.org/0000-0002-0695-8215 Emad Abdelrehim Taha https://orcid.org/0000-0001-6549-2526}

\section{Supplementary material}

Supplementary material can be found via https://doi.org/10.5653/ cerm.2020.03965.

\section{References}

1. Pereira N, Cozzubbo T, Cheung S, Palermo GD. Lessons learned in andrology: from intracytoplasmic sperm injection and beyond. Andrology 2016;4:757-60.

2. Palermo GD, Neri QV, Rosenwaks Z. To ICSI or not to ICSI. Semin Reprod Med 2015;33:92-102.

3. Borges E Jr, Braga DP, Provenza RR, Figueira RC, laconelli A Jr, Setti AS. Paternal lifestyle factors in relation to semen quality and in vitro reproductive outcomes. Andrologia 2018;50:e13090.

4. Simon L, Brunborg G, Stevenson M, Lutton D, McManus J, Lewis SE. Clinical significance of sperm DNA damage in assisted reproduction outcome. Hum Reprod 2010;25:1594-608.

5. Sun TC, Zhang Y, Li HT, Liu XM, Yi DX, Tian L, et al. Sperm DNA fragmentation index, as measured by sperm chromatin dispersion, might not predict assisted reproductive outcome. Taiwan J Obstet Gynecol 2018;57:493-8.

6. Green KA, Patounakis G, Dougherty MP, Werner MD, Scott RT Jr, Franasiak JM. Sperm DNA fragmentation on the day of fertilization is not associated with embryologic or clinical outcomes after IVF/ICSI. J Assist Reprod Genet 2020;37:71-6.

7. Zanetti BF, Braga DP, Setti AS, laconelli A Jr, Borges E Jr. Predictive factors for biochemical pregnancy in intracytoplasmic sperm injection cycles. Reprod Biol 2019;19:55-60.

8. Polat M, Bozdag G, Yarali H. Best protocol for controlled ovarian hyperstimulation in assisted reproductive technologies: fact or opinion? Semin Reprod Med 2014;32:262-71.

9. World Health Organization. WHO Laboratory manual for the examination and processing of human semen. Geneva: World Health Organization; 2010.

10. Palermo GD, Neri QV, Schlegel PN, Rosenwaks Z. Intracytoplasmic sperm injection (ICSI) in extreme cases of male infertility. PLoS One 2014;9:e113671.

11. Martinez-Soto JC, de DiosHourcade J, Gutierrez-Adan A, Landeras $\mathrm{J}$, Gadea J. Effect of genistein supplementation of thawing medium on characteristics of frozen human spermatozoa. Asian $J$ Androl 2010;12:431-41.

12. Al Omrani B, Al Eisa N, Javed M, Al Ghedan M, Al Matrafi H, Al Sufyan $\mathrm{H}$. Associations of sperm DNA fragmentation with lifestyle factors and semen parameters of Saudi men and its impact on ICSI outcome. Reprod Biol Endocrinol 2018;16:49.

13. ALPHA Scientists In Reproductive Medicine; ESHRE Special Interest Group. Istanbul consensus workshop on embryo assessment: proceedings of an expert meeting. Reprod Biomed Online 2011; 22:632-46.

14. Alvarez Sedo C, Bilinski M, Lorenzi D, Uriondo H, Noblia F, Longobucco V, et al. Effect of sperm DNA fragmentation on embryo development: clinical and biological aspects. JBRA Assist Reprod 2017:21:343-50.

15. Aggarwal A, Puri M, Dada R, Saurabh G. Correlation between leukocytospermia and oxidative stress in male partners of infertile couples with leukocytospermia. Int J Reprod Contracept Obstet Gynecol 2015;4:168-72.

16. Charehjooy N, Najafi MH, Tavalaee M, Deemeh MR, Azadi L, Shiravi $\mathrm{AH}$, et al. Selection of sperm based on hypo-osmotic swelling may improve ICSI outcome: a preliminary prospective clinical trial. Int J Fertil Steril 2014;8:21-8.

17. Cincik M, Ergur AR, Tutuncu L, Muhcu M, Kilic M, Balaban B, et al. Combination of hypoosmotic swelling/eosin $Y$ test for sperm membrane integrity evaluation: correlations with other sperm parameters to predict ICSI cycles. Arch Androl 2007;53:25-8.

18. Evgeni E, Charalabopoulos K, Asimakopoulos B. Human sperm DNA fragmentation and its correlation with conventional semen parameters. J Reprod Infertil 2014;15:2-14.

19. Pocate-Cheriet K, Heilikman I, Porcher R, Barraud-Lange V, Sermondade $\mathrm{N}$, Herbemont $\mathrm{C}$, et al. Predicting the clinical outcome of ICSI by sperm head vacuole examination. Syst Biol Reprod Med 2017:63:29-36.

20. Bradley CK, McArthur SJ, Gee AJ, Weiss KA, Schmidt U, Toogood L. Intervention improves assisted conception intracytoplasmic sperm injection outcomes for patients with high levels of sperm DNA fragmentation: a retrospective analysis. Andrology 2016; 4:903-10. 
21. Li Z, Wang L, Cai J, Huang H. Correlation of sperm DNA damage with IVF and ICSI outcomes: a systematic review and meta-analysis. J Assist Reprod Genet 2006;23:367-76.

22. Santos R, Palos-Ladeiro M, Besnard A, Porcher JM, Bony S, Sanchez W, et al. Relationship between DNA damage in sperm after ex vivo exposure and abnormal embryo development in the progeny of the three-spined stickleback. Reprod Toxicol 2013;36:6-11.

23. Xue LT, Wang RX, He B, Mo WY, Huang L, Wang SK, et al. Effect of sperm DNA fragmentation on clinical outcomes for Chinese couples undergoing in vitro fertilization or intracytoplasmic sperm injection. J Int Med Res 2016;44:1283-91.

24. Osman A, Alsomait $H$, Seshadri S, El-Toukhy T, Khalaf Y. The effect of sperm DNA fragmentation on live birth rate after IVF or ICSI: a systematic review and meta-analysis. Reprod Biomed Online 2015;30:120-7.
25. Gat I, Tang K, Quach K, Kuznyetsov V, Antes R, Filice M, et al. Sperm DNA fragmentation index does not correlate with blastocyst aneuploidy or morphological grading. PLoS One 2017;12:e0179002.

26. Zini A, Boman JM, Belzile E, Ciampi A. Sperm DNA damage is associated with an increased risk of pregnancy loss after IVF and ICSI: systematic review and meta-analysis. Hum Reprod 2008;23: 2663-8.

27. Robinson L, Gallos ID, Conner SJ, Rajkhowa M, Miller D, Lewis S, et al. The effect of sperm DNA fragmentation on miscarriage rates: a systematic review and meta-analysis. Hum Reprod 2012;27: 2908-17.

28. Zhang Z, Zhu L, Jiang H, Chen $H$, Chen Y, Dai Y. Sperm DNA fragmentation index and pregnancy outcome after IVF or ICSI: a meta-analysis. J Assist Reprod Genet 2015;32:17-26. 